\title{
Una prospettiva individualizzata sulla religione antica
}

Ancient Religion: An Individualized Perspective

Jörg Rüpke

\section{OpenEdition}

1 Journals

\section{Edizione digitale}

URL: http://journals.openedition.org/mythos/659

DOI: $10.4000 /$ mythos. 659

ISSN: 2037-7746

\section{Editore}

Salvatore Sciascia Editore

\section{Edizione cartacea}

Data di pubblicazione: 1 décembre 2017

Paginazione: 145-155

ISSN: $1972-2516$

\section{Notizia bibliografica digitale}

Jörg Rüpke, « Una prospettiva individualizzata sulla religione antica », Mythos [Online], 11 | 2017, online dal 24 septembre 2019, consultato il 28 septembre 2019. URL : http://journals.openedition.org/ mythos/659; DOI : 10.4000/mythos.659 


\section{Una prospettiva individualizzata sulla religione antica}

\section{Jörg Rüpke}

\begin{abstract}
Riassunto
La religione dell'antichità, ma anche quella dei periodi successivi, è stata spesso studiata in rapporto alla sua forma e funzione sociale, ora come "polis religion" (religione civica), ora per "gruppi" o "comunità religiose". Ebbene, gli studi che ho recentemente condotto sulla devianza religiosa suggeriscono come le pratiche religiose dell'antichità, così come le concettualizzazioni degli antichi in merito alle questioni religiose, consentano di andare ben al di là di questo approccio. In questa sede, tenterò di inserire i dati in una cornice interpretativa più ampia. Sulla scia del recente dibattito sulla struttura e l'agency individuale nelle scienze sociali - ovvero la capacità di agire di un soggetto, ma al tempo stesso la responsabilità assegnata dagli altri al soggetto stesso -, l'ipotesi che intendo sostenere è che la religione può più proficuamente essere interpretata come una modalità di agire, benché dotata di un certo potenziale di rischio. La religione, infatti, attribuisce un'agency a soggetti umani o sovrumani (come per esempio gli dèi), la cui importanza può anche essere messa in discussione. Questo approccio tuttavia consente di analizzare meglio le trasformazioni religiose del mondo antico. È una prospettiva che non si limita unicamente ad affrontare la "religiosità" individuale, collocata ai margini della religione organizzata, che può essere definita in termini di devianza; ma offre anche una base di partenza per uno sguardo più attento ai processi di costruzione e istituzionalizzazione dei gruppi religiosi. In questo quadro interpretativo, la religione antica cessa di rappresentare una categoria differente rispetto a quelle moderne, considerate prevalentemente religioni private e individuali.
\end{abstract}

\section{Parole chiave}

Roma • religione civica • individualizzazione

\section{Keywords}

Rome - polis religion - individualisation

\begin{abstract}
For antiquity and beyond religion has been seen through its social form and function, be it „polis religion" or more intimate religious groups and "communities“. My studies on religious deviance have shown that ancient religious practices as well as ancient conceptualisations of religion can go beyond this. Here, an attempt to situate these findings in a larger framework will be made. On the basis of recent discussion about structure and individual agency in the social sciences I will argue that for many purposes and in particular for observing religious change in antiquity, religion can fruitfully be regarded as a risky form of acting that attributes agency to agents the relevance of whom might be questioned by others present in the situation. Such a perspective is not just addressing individual „religiosity“ at the margins of organized religion ("deviance"), but offering a starting point for a fresh look onto processes of grouping and institutionalization. Also, in such a perspective ancient religion cannot any longer be seen as categorically different from „modern“ supposedly privatized and individualized religion.
\end{abstract}

\section{Introduzione}

Tome si definisce la religione in relazione all'individuo? Questa domanda fondamentale è alla base di ogni approccio che miri a occuparsi dell'agency individuale in contesti religiosi, sia che si prendano in considerazione i sacra publica celebrati da una sedicente élite sia quelli di un gruppo di devoti di Iside o dei promotori di un culto locale dell'imperatore.

1 *Desidero ringraziare Daniela Bonanno per la traduzione del testo e per il cortese invito presso l'Università degli Studi di Palermo che, insieme a Nicola Cusumano, ha voluto rivolgermi nell'ambito degli incontri del Dottorato "Filologia e Cultura greco-latina e Storia del Mediterraneo" e "Studi letterari, filologici-linguistici e storico-cul- 
Il paradigma della "religione di gruppo" è servito a definire una vasta gamma di pratiche che non rientravano nell'ambito della religione pubblica o che erano da questa coordinate in modo molto limitato ${ }^{2}$. Il concetto presuppone il ruolo degli individui nella cooperazione religiosa e nella formazione dei gruppi. Per quanto mi riguarda, esaminerò il problema dal punto di vista teorico, proponendo un modello analitico di religione, intesa come risorsa a disposizione dell'individuo, capace di incrementare la sua capacità di agire, di rafforzarne l'identità e di sostenere l'efficacia della sua comunicazione con il divino. Questo contributo dunque farà leva tanto sulle ricerche recenti in ambito psicologico e sociale, quanto sulla discussione relativa al concetto stesso di religione nell'ambito specifico della storia delle religioni. Ponendo l'accento sull'agente individuale nella nozione di religione, in riferimento all'agency, all'identità collettiva e personale, e alla comunicazione, questo saggio sosterrà l'urgenza di superare le nozioni di "religione civica", di "culti" e "comunità"; e di storicizzare i fenomeni descritti in tali termini, nel tentativo di porre nuovi interrogativi, senza necessariamente suggerire soluzioni, come invece accade nel caso dell'approccio che prende le mosse dai culti o dalla religione civica.

A fare da sfondo intellettuale a questa mia riflessione è il lavoro condotto nell'ambito del Gruppo di ricerca "Religious Individualisation in Historical Perspective" del Max-Weber-Kolleg dell'Università di Erfurt e la critica elaborata al suo interno sulla diffusa pratica di assegnare, in modo dicotomico, un'agency religiosa individuale alla modernità, considerando invece, almeno per quel che riguarda la religione, il mondo antico come spazio privilegiato dell'agire collettivo. Alla base di tale posizione oggetto della nostra critica, si trova una definizione precisa di religione. Sulla scia degli studi del sociologo francese Émile Durkheim (1858-1917), la religione è stata, infatti, definita come un'impresa collettiva (una definizione basata peraltro non sulle società semplici, ma sul modello delle chiese cristiane) e dunque, frequentemente, criticata per la sua incapacità di tenere adeguatamente in conto gli sviluppi religiosi non occidentali.

Se viceversa si assume come orizzonte metodologico la concettualizzazione della religione a partire dall'individuo e del suo contesto sociale, allora ad essere oggetto di tale strategia di ricerca non saranno più i sistemi di credenze o di pratiche rituali elaborati dagli osservatori interni ed esterni. Tali sistemi, infatti, possono essere oggetto di "appropriazione" da parte dell'individuo solo in modo parziale e imperfetto ${ }^{3}$. Per appropriazione, si intende quel processo in base al quale i contenuti della tradizione accolta vengono contemporaneamente selezionati, modificati e variamente riproposti nel contesto sociale da parte dei singoli.

A essere sotto esame dunque sarà la "religione vissuta" dell'Antichità, nelle sue varianti individuali, nelle diverse situazioni e costellazioni sociali ${ }^{4}$. Solo raramente tali interazioni si concretizzano in network, organizzazioni o testi scritti che possono svilupparsi autonomamente e quindi essere assimilati a quelle che oggi chiamiamo "religioni".

Come definire allora la religione? Dove trovarla nell'individuo, se non si volge comunque

turali". Questo contributo si inserisce nell'ambito del progetto "Lived Ancient Religion" (ERC Advanced Grant dell'Unione Europea, $7^{\text {th }}$ Framework Program 2008-2013, grant agreement no. 295555). Ringrazio inoltre le colleghe e i colleghi del Kolleg-Forschergruppe "Religious Individualisation in Historical Perspective" del Centro Max Weber, Università di Erfurt (DFG FOR 1013).

2 Vedi RüPKE 2007 e RÜPKE 2015.

3 Per il concetto di appropriazione, vedi de Certeau 1984, de Certeau 2007 e Füssel 2006; secondo Certeau 1987, l'imperfezione è un aspetto costitutivo del processo di appropriazione.

4 RÜPKE 2012 e MCGUIRE 2008. 
lo sguardo alla società e ai suoi fenomeni collettivi? Come concettualizzarla, se l'individuo non è isolato dalla società? Prendendo le mosse da un approccio fenomenologico, suggerisco di analizzare il problema, a partire da tre prospettive differenti:

1. L'individuazione dei contesti in cui la religione rafforza l'agency individuale di un uomo o di una donna; la loro competenza e creatività nell'affrontare problemi quotidiani o straordinari;

2. Il contributo della religione alla costruzione individuale dell'identità collettiva che rende l'azione o il pensiero individuale, parte di un gruppo o di compagini sociali di forma e complessità differenti;

3. Il ruolo della religione nella comunicazione interpersonale, ovvero la ricostruzione delle modalità con cui la religione strategicamente è chiamata in causa e impiegata come mezzo di comunicazione, capace a sua volta di dar luogo a ulteriori forme di comunicazione.

La prospettiva che ho scelto è quindi quella dell'analisi della religione a partire dall'agente. Spero quindi di contribuire alla comprensione dell'efficacia della religione in contesti più ampi, dalle istanze individuali di appropriazione alla costruzione religiosa sottesa, per esempio, alla semantica capitolina, mitraica, isiaca, giudaica fino a quella che mi piace chiamare "religione vissuta". Per una migliore comprensione della religione tout court è opportuno tenere presente e valorizzare tutto il materiale pertinente, evitando così di tracciare distinzioni troppo rigide tra „culti "ufficiali”, come per esempio quello "capitolino”, o culti “elettivi”, per esempio quelli di Silvano o di Cerere.

\section{Azione religiosa}

La sociologia interpretativa e l'antropologia culturale hanno inteso il comportamento umano come un'azione che deve essere interpretata sullo sfondo di significati socialmente prodot$\mathrm{ti}^{5}$. L'approccio dei sociologi americani a partire dagli anni Venti del XX secolo, conosciuto come "Pragmatismo", ha consentito di perfezionare queste analisi. Azione è soprattutto agire per risolvere problemi. Frequentemente l'individuo si trova di fronte a situazioni che non possono essere affrontate semplicemente in modo prestabilito, ricorrendo a obiettivi e significati preordinati. È proprio nel corso dell'azione, sviluppata in modo da risolvere un problema pressante, che obiettivi e significati vengono sviluppati e modificati, poiché la persona che agisce è sempre parte di un contesto sociale che comprende altri agenti e tradizioni procedurali. Nell'ambito di un concreto e casuale spazio di possibilità, l'agente può sviluppare soluzioni inedite; può quindi essere creativo ${ }^{6}$.

In un articolo dal titolo "What is agency", Mustafa Emirbayer e Ann Mische hanno delineato brevemente questa teoria e hanno reinserito il concetto di agency in questo contesto ${ }^{7}$. Benché nelle loro riflessioni questi studiosi non prendano specificatamente in considerazione la religione, tuttavia si deve loro l'introduzione della nozione di tempo, nella teoria dell'azione, che è particolarmente utile per un concetto di religione centrata sull'attore individuale. Nella loro definizione, "agency" è "the temporally constructed engagement by actors of different

5 Questo è vero per Clifford Geertz come per Max Weber: cfr. SEgal 1999.

6 Cfr. JOAS 1996.

7 Emirbayer, Mische 1998 (sulle teorie brevemente passate in rassegna in questo articolo, cfr. 964-968). 
structural environments - the temporal-relational contexts of action - which, through the interplay of habit, imagination, and judgment, both reproduces and transforms those structures in interactive response to the problems posed by changing historical situations ${ }^{8}{ }$.

L'agency è quindi definita e sviluppata su tre livelli temporali:

a) rispetto ad azioni passate, e in riferimento a routine stabilite, vengono sviluppate "schematizzazioni" sempre più elaborate ed efficaci - per esempio: "ho imparato che è opportuno cominciare un seminario ringraziando gli organizzatori";

b) in relazione a conseguenze future dell'azione del singolo e della loro proiezione sul futuro, sono sviluppate ipotesi su scala sempre più ampia - per esempio: "sono convinto che sia meglio presentare una tesi rischiosa per favorire il progresso scientifico";

c) in relazione alla valutazione della situazione presente, per quel che riguarda la realizzabilità e una valutazione all'interno del contesto sociale, maggiori esperienze sociali sviluppano forme di "contestualizzazione" sempre più adeguate - per esempio: "è importante avere presente che l'uditorio non è limitato ai soli organizzatori del seminario"".

Non è l'individuo che possiede un'agency tout court, ma costui agisce in quanto possessore di un'agency, avendo a che fare con il contesto strutturale in una situazione data: "gli attori si impegnano, in relazione alla loro agency, con i loro contesti di strutturazione. Struttura e agency si costruiscono quindi reciprocamente ${ }^{10}$.

Quali sono le implicazioni di questa riflessione per l'azione religiosa? Illustrerò brevemente qualche fenomeno al centro di questa linea di ricerca.

Per lo sviluppo di schematizzazioni, al di là delle esperienze passate, le proprie e quelle degli altri, uno storico delle religioni deve chiedersi come l'agire religioso è appreso e applicato in situazioni specifiche. Quali sono i contesti di apprendimento religioso? Come interagiscono osservazione, partecipazione limitata o assistita, insegnamento formale o informale? Come imparano gli individui a interpretare le esperienze come religiose? Come possono sviluppare ruoli religiosi nuovi o assumere identità religiose personali? Solitamente abbiamo a che fare con micro-tradizioni locali: sequenze rituali da riprodurre, volontà di distinguersi, di adeguarsi, o di seguire una moda. Esempi privilegiati sono i cimiteri, le tavolette votive, l'uso di ornamenti in una processione.

Proiettarsi nel futuro necessita, invece, di strutture temporali che possono essere usate per dare un ordine proprio al futuro. Chi introduce nuove feste o chi riforma il calendario religioso? Processi di cambiamento nelle istituzioni, come per esempio la creazione di nuovi sacerdozi o la loro trasformazione, possono essere esaminati in termini di agency. Non si tratta di sviluppi naturali: tali processi hanno, infatti, alla base un'iniziativa personale o talora un mero fraintendimento.

L'interpretazione dell'esperienza sociale per giudicare le possibilità di una situazione, che si è appena presentata e che può conoscere ulteriori sviluppi, offre grande spazio all'azione religiosa. Ritmi temporali possono essere cambiati dalle pratiche di sacralizzazione; il luogo dell'azione può subire trasformazioni, grazie all'azione rituale locale, per affrontare problemi diversi fra loro e separati anche dal punto di vista spaziale; competenze religiose, per esempio, di specialisti religiosi possono essere trasferite o derogate in base a nuovi argomenti di legitti-

8 Emirbayer, Mische 1998, 970.

9 EMirbayer, Mische 1998, 975, 983, 993.

10 Emirbayer, Mische 1998, 1004. 
mità religiosa; decisioni politiche possono essere influenzate chiamando in causa gli oracoli ${ }^{11}$.

Riassumendo, questo allargamento del contesto definito rilevante per momenti critici, tramite l'introduzione di attori o istanze divine, è tipico della religione. Più probabilmente, è questa strategia che apre nuovi spazi all'immaginazione e a interventi individuali creativi, incrementando quindi l'agency ${ }^{12}$. Ma è al tempo stesso questo stesso meccanismo che può seriamente diminuire l'agency riservata agli attori religiosi.

\section{Identità religiose}

Il concetto di identità collettiva è stato giustamente criticato, laddove interpretato come senso di consapevolezza permanente, o addirittura esclusiva, di appartenenza e come rappresentazione del singolo, quale membro di quelle forme di adesione che si assegnano in modo definitivo all'interno di determinati gruppi sociali ${ }^{\mathbf{1 3}}$.

Dati gli effetti empiricamente verificati sul comportamento individuale di una seppur vaga forma di appartenenza ${ }^{14}$, resta difficile fare a meno di questo concetto. Nel tentativo di sviluppare una nozione di religione empiricamente ampia (che copra cioè una vasta gamma di fenomeni), è utile allargare la prospettiva all'azione compresa nel concetto di agency, rispetto a quello di "identità collettiva". Allo stesso tempo, è necessario avere presente una nozione differenziata e dinamica di tali identità. Le ricerche più recenti, nell'ambito della psicologia sociale, hanno fatto diversi tentativi in questo senso ${ }^{15}$. Per quanto mi riguarda seguirò la via suggerita da tre psicologi di New York che propongono uno schema composto da sette fattori, per comprendere pienamente e distintamente i diversi aspetti del fenomeno. Nel loro sforzo di concettualizzazione, la religione non ha alcuna rilevanza. Tutti i fattori sono concettualizzati a livello individuale e non in relazione al gruppo: per esempio, "io possiedo un'identità collettiva anche se questa collettività non esiste mai”.

Gli elementi enumerati da Ashmore, Deaux e McLaughlin-Volpe sono i seguenti: autoclassificazione; valutazione del grado di adesione (qualunque sia la forma) da parte di individui e della loro percezione dei giudizi degli altri; importanza attribuita a questo particolare tipo di adesione; l'attaccamento, cioè il coinvolgimento emotivo e il senso di interdipendenza (che potenzialmente ricade in un'ampia sovrapposizione di identità personale e collettiva); “il grado di integrazione di una determinata identità collettiva, nel procedere relazionale della vita quotidiana della persona" (social embeddedness); l'influenza di una determinata identità collettiva sul comportamento individuale; e infine tutta la dimensione cognitiva dell'immaginario e dei racconti relativi ai valori, alle caratteristiche e alla storia di un gruppo rilevante, per come è conosciuto e percepito dall'individuo stesso - che sia, ancora una volta, di un gruppo reale o immaginato ${ }^{16}$. Considerata la critica verso i diversi concetti di "religione", è importante sottolineare che la nozione di "gruppo" non coinvolge ogni associazione organizzata, ma si riferisce a raggruppamenti contingenti di attori (non necessariamente umani), in cui l'individuo inse-

11 Cfr. Emirbayer, Mische 1998, 1000-1002.

12 Cfr. le ipotesi in Emirbayer, Mische 1998, 1006-1007.

13 Cfr. Rebillard 2012, che opta per il concetto di "salient identity".

14 A proposito della “teoria dell'identità sociale", si vedano TAJfel 1974 (69 per la definizione di "gruppo") e Turner 1975. Per una sintesi Ellemers, Spears, Doosje 1999.

15 Per esempio Cameron 2004, 241, che individua tre fattori di centralità cognitiva per l'agente individuale, tra cui le associazioni emotive e il senso del dovere nei confronti dei membri dello stesso gruppo.

16 Ashmore, Deaux, McLaughlin-Volpe 2004, 83 con una tavola riassuntiva. 
risce se stesso, o se stessa, o da cui prende le distanze. Noi immaginiamo essere parte di molti gruppi diversi nelle diverse situazioni quotidiane.

Naturalmente tutto questo può condurre alla formazione di identità collettive sempre più complesse e di diverse forme di appartenenza, come di prese di distanza ${ }^{17}$. In che modo la religione (che verrà più precisamente definita nell'ultima parte di questo saggio) sia coinvolta in un particolare contesto storico e culturale e come possa essersi trasformata nel tempo o nei diversi processi di embricazione, è esattamente l'oggetto della mia analisi. Gli studiosi di religione dovrebbero essere interessati tanto alle identità familiari rispetto ai gruppi sociali primari, come a quelli secondari. Si occuperanno anche dei diversi ruoli delle identità locali, regionali e transregionali come di transfer e di interferenze tra questi livelli ${ }^{18}$. È di grande importanza evitare di considerare questi gruppi o associazioni come essenziali. Sulla scia di Pierre Bourdieu, gli archeologi, concentrandosi su resti materiali, hanno messo in guardia su ogni diretta interferenza da parte di oggetti materiali di rigide relazioni sociali. Una comunità particolare non è data, ma sempre creata in relazione alle azioni che tale comunità vuole produrre ${ }^{19}$. Si possono concettualizzare le identità religiose come particolari cornici situazionali ${ }^{20} \mathrm{e}$ perciò integrarle nel modello teorico di azione dell'agency.

Allo stesso modo, l'agire può essere descritto come un esito contingente delle identità ${ }^{21}$.

Tuttavia, per differenziarle una dall'altra, le dinamiche e la struttura diacronica del concetto di "agency" possono essere combinate con l'analisi sincronica della struttura orizzontale del contesto sociale. Infatti, si attribuisce importanza a entrambi i poli. Infine, sostituendo un concetto ridotto all'essenziale di "religioni” (al plurale) con un complesso di identità collettive e individuali, si è in grado di analizzare i processi di formazione dei gruppi religiosi nei loro differenti percorsi e nei loro mutevoli punti di forza: per esempio, i partecipanti ad un rituale cristiano; gli Ebrei il sabato; Romani in quanto decuriones; Efesini durante una processione per l'imperatore nel III secolo. Solamente il lavoro ininterrotto degli intellettuali e il conferimento di diritti ai vescovi da parte di Costantino ha creato uno o, meglio, alcune organizzazioni che rafforzarono i codici di comportamento cristiano per i suoi devoti durante i secoli seguenti.

\section{Comunicazione religiosa}

Prendendo ora in considerazione, all'interno di questo percorso, il concetto di comunicazione, vedremo la nozione di religione arricchirsi di altri aspetti che concorrono a fornirne una descrizione più ampia. Il primo aspetto, innanzitutto, è che la comunicazione è opera degli individui, che agiscono come comunicatori o come parte di un pubblico. Anche nell'ambito della comunicazione di massa le reazioni di coloro cui essa è rivolta possono variare ampiamente e, anche se il messaggio ha raggiunto i suoi destinatari ed è stato compreso, può essere interpretato e giudicato in modo differente ${ }^{22}$.

La teoria semantica classica della comunicazione prende le mosse proprio dalla relazione tra il mittente e il destinatario. La persona che invia il messaggio inizia ad agire come una fonte.

17 Cfr. Ashmore, Deaux \& McLaughlin-Volpe 2004, 84.

18 Cfr. Jones 2012 a proposito delle identità "greche" antiche.

19 VAN DOMMELEN et al. 2005.

20 Cfr. Emirbayer, Mische 1998, 992, che mettono in evidenza il carattere frequentemente retrospettivo del concetto di identità.

21 Ashmore, Deaux, Mclaughlin-Volpe 2004, 101-104.

22 Cfr. Chandler 2011, s.v. "active audience theory". 
Un segnale è trasmesso al destinatario e ricevuto come informazione, comando o simili. Ogni elaborazione del modello si concentrava sui processi di codifica e decodifica cui era destinato il messaggio o sul contesto sociale, lo scenario dell'atto comunicativo, inteso lato o stricto sensu. Ogni forma di comunicazione basata sui cosiddetti media primari (lingua, linguaggio del corpo, gesti impiegati nella comunicazione faccia a faccia tra mittente e destinatario) è già ricca di interazione. Viceversa, ogni interazione implica comunicazione. Nel caso estremo di un'interazione simbolica, l'azione è determinata dall'intenzione di trasmettere un messaggio, benché altamente criptato.

La teoria della pertinenza (relevance theory), elaborata da Dan Sperber e Deirdre Wilson negli anni Ottanta, ha integrato la complessità della comunicazione umana in un modello basato sul principio che "i processi cognitivi sono orientati alla pertinenza", partendo dal presupposto che le attività di codifica e di decodifica sono implicate nell'uso elementare del linguaggio ${ }^{23}$. Si agisce sulla base degli stimoli più pertinenti del contesto. Lo stesso vale per la comunicazione. Nella comunicazione, l'intenzione del mittente di rendere i suoi punti di vista chiari, o più chiari, a un'audience procede insieme all'esigenza di rendere questa intenzione informativa reciprocamente compresa. L’obiettivo può essere garantito dagli stimoli che chiariscono questa intenzione; quest'ultimo aspetto prende il nome di "comunicazione ostensivo-inferenziale" 24 . Il risultato della comunicazione è la reciproca trasformazione dell'ambiente. ${ }^{25}$ Dati i tanti stimoli che l'uditorio potenziale riceve, lo stimolo ostensivo deve rispondere all'aspettativa di una perfetta pertinenza della comunicazione che ne seguirà. $\mathrm{O}$ più precisamente, lo sforzo necessario per processare l'informazione (intesa in senso lato) deve essere proficuo per l'uditorio. Il comunicatore deve produrre l'informazione più pertinente che vuole ed è in grado di produrre ${ }^{26}$.

Comprensione significa quindi costruire simultaneamente ipotesi su un contenuto esplicito, su assunti legati al contesto (cioè le premesse) e sulle ripercussioni sul contesto (le conclusioni) ${ }^{27}$

Non intendo occuparmi della capacità del modello di spiegare gli artifici retorici, l'ironia o il mentire. Tornando ora alla comunicazione religiosa - a quanto mi risulta - essa non è stata finora analizzata nell'ambito di questa cornice. La presenza frequente di personale religioso (sacerdoti, indovini, individui giovani e anziani) solleva il problema della "competenza" religiosa, un potenziale paradigma per agency e potere, per la sua distribuzione e limitazione. La concreta presenza dei media agisce come stimolo ulteriore e allarga la prospettiva diadica della comunicazione umano-divina a destinatari secondari, audiences e testimoni, esperti e turisti. La scelta di media o di "stimoli" costituisce questi insiemi. Infatti, la preghiera pronunciata, la maledizione scritta, il sacrificio familiare e le processioni pubbliche costruiscono un pubblico volta per volta differente ${ }^{28}$. Certe forme di comunicazione con il divino possono essere monopolizzate, come dimostra la normativa contro gli indovini in età tardo-antica ${ }^{29}$. Sullo sfondo dell'attenzione continua al divino, è importante costruire il carattere precario della disponibilità degli attori divini.

23 Sperber, Wilson 1987, 700.

24 SPERber, Wilson 1987, 700.

25 Sperber, Wilson 1987, 699.

26 Wilson, SPERBER 2002, 257-258.

27 Wilson, Sperber 2002, 262.

28 A proposito delle dimensioni del concetto di pubblico in una prospettiva storico-religiosa, si vedano GLADIGOW 1995; Rüpke 1995, 605-628; Fine 2010; Mullaney, Vanhaelen, Ward 2010 e Wolson, Yachnin 2010.

29 Cfr. FögEN 1993. 
La comunicazione non può essere interpretata senza fare riferimento al potere e alle diseguaglianze sociali, senza spostare il focus sulla struttura sociale. Ciò si aggiunge all'approccio centrato sull'attore che proponiamo in questa sede e ci riconduce alle specificità della teoria della pertinenza. Se la pragmatica della comunicazione sollecita stimoli specifici e straordinari per guadagnare il riconoscimento degli dèi, l'introduzione della comunicazione religiosa nella comunicazione ordinaria produce ugualmente uno stimolo eccezionale. La pertinenza straordinaria è sostenuta dall'introduzione delle divinità nel contesto interumano, della comunicazione interpersonale e ne altera le regole. Le diverse formule fisse dei media della comunicazione religiosa attestano la loro importanza, la loro sopravvivenza, il successo di questo genere di comunicazione. Aiutano a impiegare, a ripetere facilmente o introdurre in modo ricorsivo gli elementi religiosi nell'azione dell'agente o dell'identità collettiva.

\section{Dal concetto di religione alla teoria della religione}

Agency, identità collettiva e comunicazione definiscono le tre prospettive che ho appena impiegato per porre il focus sull'individuo nella sua socialità e nel suo ambiente. Ciascuno dei tre termini si riferisce a individui che cercano di comunicare:

- agendo sul loro contesto come esseri temporali, nella loro percezione del passato, del presente e del futuro (agency);

- ponendo il loro sé strutturato in un contesto altrettanto socialmente strutturato (identità);

- volgendosi al loro ambito sociale in modo interattivo, in forma diretta, attraverso intermediari o altro tipo di media (comunicazione).

Questi tre aspetti concorrono a spiegare il concetto di una cultura basata sull'individuo ${ }^{30}$. Tale descrizione fenomenologica degli individui ruota su un concetto di religione definito solo in base a specificità religiose e su queste ultime è incentrata. Riguardo a questo punto, torno alla tecnica delle definizioni sostanziali. Quindi definisco la religione come ampliamento del contesto situazionale rilevante al di là del contesto sociale immediato e plausibile degli essere umani (e frequentemente anche degli animali). Questo ampliamento è realizzato attraverso forme di azione dell'agente specificamente religioso, nella comunicazione e nella formulazione dell'identità collettiva e personale. Ciò che possiamo qualificare come "non immediatamente plausibile" varia da cultura a cultura e persino da situazione a situazione. La "plausibilità", ovvero la capacità di ottenere l'assenso degli altri, è di per sé una categoria comunicativa o, meglio, retorica.

Parenti defunti, esseri divini concettualizzati come persone, luoghi che non possono essere identificati topograficamente, popoli al di là dell'oceano - tutto questo ricade in quella zona grigia identificata come religione. Ciò che culturalmente può essere messo in discussione è ovviamente un'interpretazione in base alla quale l'osservatore, lo studioso di religione è parzialmente (ma di solito esclusivamente responsabile). L'enfasi eccessiva sulle "divinità" o sulla distinzione tra "religione" e "magia" ${ }^{1}$ sono esempi dei fraintendimenti che ne derivano.

Per gli obiettivi di uno storico delle religioni non è necessario, ma è utile sviluppare un concetto di religione, che dia forma a racconti di trasformazioni religiose, in una teoria che cerca ancora di "spiegare" la religione.

30 Cfr. per esempio Swidler 1986, 277.

31 A questo proposito Отто 2011. 
L'atto di costruzione di agenti presumibilmente rilevanti, ma inizialmente meno plausibili (o "controintuitivi", nella terminologia delle teorie evoluzionistiche della religione), sembra produrre continuamente un surplus di autostabilizzazione, di potere o di capacità di risolvere i problemi. Tale atto diventa tuttavia immediatamente oggetto di contestazione a causa dell'iniquità prodotta dal successo di questa operazione. Forme di sacralizzazione nell'ambito di un indiscusso plausibile ed evidente contesto sono elementi di una simile azione strategica.

La metafora dell'"investimento" che ho impiegato senza difficoltà si riferisce al lusso materiale, alle spese enormi della religione per i media. E include le immagini di culto e i santuari, i rituali complessi, le strategie testuali e comunicative, per non parlare del personale. Qui, un vasto campo di teorie dei media e della ricerca sulla comunicazione può essere messa a disposizione degli storici per esigenze euristiche, come degli scienziati sociali per test empirici. Lo stesso vale per le indagini su quegli individui che si trovano ad affrontare situazioni di inferiorità religiosa. Seguendo la teoria dell'identità sociale, tali individui possono optare nell'ambito di un contesto religioso per strategie di trasformazione sociale o possono decidere di lasciare questo contesto religioso e tentare di elevarsi socialmente (oppure optare per l'immobilismo, rinunciando ad ogni forma di agency) $)^{32}$.

Se la religione è una risorsa per ampliare l'agency dell'individuo, per migliorare la comunicazione e rimettere a fuoco l'identità (riducendone o aumentandone la complessità), ciò avviene in modo più congeniale per alcuni che per altri e in alcune o tutte le prospettive esaminate.

Aggiungerei che ciò vale anche nella vecchia buona abitudine dell'ateismo metodologico, tanto che la risorsa sia data o creata. Alcuni se ne avvalgono per arrogarsi una posizione che altrimenti non sarebbe loro assegnata; altri la usano per resistere e rifiutare le azioni che sono tenuti a fare, come l'arruolamento militare, il lavorare nei giorni di festa, sposarsi o mangiare cibi non graditi. I capifamiglia, i patroni, i latifondisti gradivano sottolineare la loro posizione dirigendo rituali di comunicazione religiosa. Le autorità politiche rafforzavano la loro posizione appropriandosi di queste forme, conferendo prestigio alla comunicazione religiosa e anche dignità alle pratiche religiose individuali, rafforzando paradossalmente l'agency di queste ultime. Certamente, la religione era, per molti versi, una religione integrata (embedded), ma senza i meccanismi della comunicazione religiosa non sarebbe riuscita a conferire nessun tipo di agency aggiuntiva.

Ovunque si fosse creato un terreno di agency religiosa, di capitale per dirla con Bourdieu, sarebbero nate contese in relazione a questo capitale, seguendo le linee del potere istituzionale o delle organizzazioni. Ma mettere insieme istituzioni e significati condivisi è secondario rispetto all'agency o solo indifferente. L'agency si acquisisce ed è attribuita nell'ambito delle strutture. Le strutture, però, cambiano.

Jörg Rüpke

Universität Erfurt Max-Weber-Kolleg für kultur- und sozialwissenschaftliche Studien

Universität Erfurt

Nordhäuser Str. 63 99089 Erfurt joerg.ruepke@uni-erfurt.de 
Bibliografia

Ashmore et al. 2004

R.D. Ashmore et al., «An Organizing Framework for Collective Identity: Articulation and Significance of Multidimensionality", Psychological Bulletin 130, 1 (2004), 80-114.

CAmeron 2004

J.E. Cameron, «A Three-Factor Model of Social Identity», Self and Identity 3,3 (2004), 239262.

de Certeau 1984

M. de Certeau, The practice of everyday life, Berkeley 1984.

de Certeau 1987

M. de Certeau, La faiblesse de croire, Paris 1987.

de Certeau 2007

M. de Certeau, Arts de faire, Paris 2007.

Chandler 2OI I

D. Chandler, $A$ dictionary of media and communication. Oxford paperback reference, Oxford 2011.

ElLEMERs et al. 1999

N. Ellemers, Social identity : context, commitment, content, Oxford 1999.

Emirbayer, Mische 1998

M. Emirbayer, A. Mische, «What Is Agency?», American Journal of Sociology 103, 4 (1998), 962-1023.

FINE 2010

G.A. Fine, «Sociology of the Local: Action and its Publics», Sociological Theory 28, 4 (2010), 355-376.

Fögen 1993

M.-T. Fögen, Die Enteignung der Wahrsager: Studien zum kaiserlichen Wissensmonopol in der Spätantike, Frankfurt a. M. 1993.

Füssel 2006

M. Füssel, «Die Kunst der Schwachen: Zum Begriff der 'Aneignung' in der Geschichtswissenschaft», Sozial.Geschichte 21, 3 (2006), 7-28.

GLADIGOW 1995

B. Gladigow, «Struktur der Öffentlichkeit und Bekenntnis in polytheistischen Religionen», in:
H.G. Kippenberg, G. Stroumsa, (eds.), Secrecy and Concealment: Studies in the History of Mediterranean and Near Eastern Religions, Leiden 1995, 17-35.

JOAS 1996

H. Joas, Die Kreativität des Handelns, Frankfurt a. M. 1996.

Jones 2012

C.P. Jones, «Joys and Sorrows of Multiple Citizenship: The Case of Dio Chrysostom", in A. Heller, A.-V. Pont (eds.), Patrie d'Origine et Patries electives: Les Citoyennetés multiples dans le monde grec d'époque romaine, Pessac 2012, 213-219.

\section{LATTE 1968}

K. Latte, «Promanteia», in K. Latte, Kurt (ed.) Kleine Schriften: zu Religion, Recht, Literatur und Sprache der Griechen und Römer, München 1968, 193-5.

McGuire 2008

M.B. McGuire, Lived Religion: Faith and Practice in Everyday Life, Oxford 2008.

Mullaney et al. 2010

S. Mullaney et al. «Religion Inside Out: Dutch House Churches and the Making of Publics in the Dutch Republic», in B. Wolson, P. Yachnin (eds.), Making Publics in Early Modern Europe: People, Things, Forms of Knowledge, Routledge Studies in Renaissance Literature and Culture, New York/London 2010, 25-36.

Отто 2011

B.-C. Otto, Magie: Rezeptions- und diskursgeschichtliche Analysen von der Antike bis zur Neuzeit, Religionsgeschichtliche Versuche und Vorarbeiten 57, Berlin 2011.

Rebillard 2012

E. Rebillard, Christians and their many identities in late antiquity, North Africa, 200-450 CE, Ithaca 2012.

RÜPKE 1995

J. Rüpke, Kalender und Öffentlichkeit: Die Geschichte der Repräsentation und religiösen Qualifkation von Zeit in Rom. RGVV 40, Berlin 1995. 
RÜPKE 2007

J. Rüpke, Gruppenreligionen im römischen Reich. Sozialformen, Grenzziehungen und Leistungen. Studien und Texte zu Antike und Christentum 43, Tübingen 2007.

\section{RÜPKE 2012}

J. Rüpke, «Lived Ancient Religion: Questioning 'Cults' and 'Polis Religion'”, Mythos ns 5 (2011) 191-204.

\section{RÜPKE 2015}

J. Rüpke, "Religious Agency, Identity, and Communication: Reflecting on History and Theory of Religion", Religion 45, 3, 344-366.

Segal 1999

R.A. Segal, «Weber and Geertz on the Meaning of Religion", Religion 29, 1 (1999), 61-71.

SPerber 1987

D. Sperber, D. Wilson, «Précis of Relevance», Behavioral \& Brain Sciences 10, 4 (1987), 697 710.

SWIDLER 1986

A. Swidler, «Culture in Action: Symbols and
Strategies", American Sociological Review 51,2 (1986), 273-286.

TAJFel 1974

H. Tajfel, «Social identity and intergroup behaviour", Social Science Information 13, 2 (1974), 65-93.

\section{TURNer 1975}

J.C. Turner, «Social comparison and social identity: Some prospects for intergroup behaviour», European Journal of Social Psychology 5 (1975), 5-34.

WiLSON, SPERBER 2002

D. Wilson, D. Sperber, «Relevance Theory», UCL Working Papers in Linguistics 13 (2002), 249-287.

Wolson, YaCHNIn 2010

B. Wolson, P. Yachnin, Paul (eds.), Making Publics in Early Modern Europe: People, Things, Forms of Knowledge, Routledge Studies in Renaissance Literature and Culture, London 2010. 